Sādhanā Vol. 38, Part 4, August 2013, pp. 621-644. (C) Indian Academy of Sciences

\title{
A novel image inpainting technique based on median diffusion
}

\author{
RAJKUMAR L BIRADAR ${ }^{1, *}$ and VINAYADATT V KOHIR ${ }^{2}$ \\ ${ }^{1} \mathrm{G}$ Narayanamma Institute of Technology and Science, Hyderabad 500008, India \\ ${ }^{2}$ Poojya Doddappa Appa College of Engineering, Gulbarga 585104, India \\ e-mail: rajkumar_lb@yahoo.com; vvkohir@yahoo.com
}

MS received 31 August 2011; revised 17 June 2012; accepted 29 May 2013

\begin{abstract}
Image inpainting is the technique of filling-in the missing regions and removing unwanted objects from an image by diffusing the pixel information from the neighbourhood pixels. Image inpainting techniques are in use over a long time for various applications like removal of scratches, restoring damaged/missing portions or removal of objects from the images, etc. In this study, we present a simple, yet unexplored (digital) image inpainting technique using median filter, one of the most popular nonlinear (order statistics) filters. The median is maximum likelihood estimate of location for the Laplacian distribution. Hence, the proposed algorithm diffuses median value of pixels from the exterior area into the inner area to be inpainted. The median filter preserves the edge which is an important property needed to inpaint edges. This technique is stable. Experimental results show remarkable improvements and works for homogeneous as well as heterogeneous background. PSNR (quantitative assessment) is used to compare inpainting results.
\end{abstract}

Keywords. Inpainting; median filter; diffusion.

\section{Introduction}

Reconstruction of missing or damaged portions of images is an ancient practice used extensively in artwork restoration. Medieval artwork started to be restored as early as the Renaissance, the motives being often as much to bring medieval pictures 'up to date' as to fill in the gaps (Walden 1985; Emile-Male 1976). Image inpainting is an image restoration problem in the form of image interpolation where a marked area is filled by its neighbourhood information. Application of image inpainting ranges from reconstruction of missing data introduced during wireless transmission to removal of cracks, scratches, noise, dust or strain marks of scanning glass on the scanned image, stamped date and red-eye from photographs and the infamous 'airbrushing'

*For correspondence 
of political enemies, etc. In the most conventional form, the user selects an area for inpainting and algorithm automatically fills-in the region with information surrounding it without loss of any perceptual quality. The concept of digital inpainting was introduced by Bertalmio et al (2000).

The proposed algorithm addresses inpainting of images with a homogeneous or heterogeneous background. The technique is based on diffusing median value of pixels which are exterior to inpainting area into the area to be inpainted. This is a simple inpainting technique which achieves better results than other inpainting techniques (Bertalmio et al 2000; Roth \& Black 2005) proposed so far.

\section{Earlier studies}

Inpainting techniques may be classified into following broad categories: (a) Partial different equations (PDE)-based algorithms; (b) Convolution filter-based algorithms; (c) Other algorithms.

Most of the inpainting work is carried out in the area of structure inpainting as it finds many real-time applications. PDE-based and convolution filter-based techniques inpaint the image through diffusion modelling. These are well-suited for structure inpainting.

\subsection{PDE-based algorithms}

Bertalmio et al (2000) have developed a digital inpainting algorithm based on the PDE and it is an extension of level lines based disocclusion method proposed by Masnou \& Morel (1998). In Bertalmio et al, inpainting the direction of arrival of isophotes is maintained by computing the direction of the largest spatial change. The direction is obtained by computing a discretized gradient vector.

Bertalmio et al (2001) used the ideas from computational fluid dynamics (CFD) to propagate isophote lines continuously from the exterior into the region to be inpainted. The main idea is to treat the image intensity as a 'stream function' for a two-dimensional incompressible flow. The Laplacian of the image intensity plays the role of the vorticity of the fluid, and it is transported into the region to be inpainted by a vector field defined by the stream function. The resulting algorithm is designed to continue isophote lines (equal gray level lines) while matching gradient vectors at the boundary of the inpainting region. The method is directly based on the NavierStokes equations for fluid dynamics.

Chan \& Shen (2000) proposed two image inpainting algorithms. Total variation (TV) inpainting model (Chan \& Shen 2000, 2001) uses an Euler Lagrange modelling. Inside the inpainting domain, this model employs anisotropic diffusion (Perona \& Malik 1990) based on contrast of the isophotes. It does not connect broken edges (i.e., single lines embedded in a uniform background). Curvature-driven diffusion (CCD) model (Chan \& Shen 2001; Chan et al 2002), is an extended TV algorithm, in which geometric information of isophotes is taken into account while defining strength of diffusion process; thus allowing the inpainting to proceed over large areas. $\mathrm{CCD}$ can connect some broken edges resulting in blurry segments. The phase transition in superconductor and Ginzburg-Landau equations (Grossauer 2003; Grossauer \& Scherzer 2003) are used to inpaint selected areas. In Tai et al (2006), normal and tangential vectors are propagated into damages/missed regions and image is reconstructed.

Telea (2004) has proposed a fast marching method (FMM) algorithm. The algorithm calculates smoothness of image from a known image neighbourhood of the pixel as a weighted 
average to inpaint. The FMM inpaints the near pixels to known region first and maintains a narrow band of pixels which separates known pixels from unknown pixels. The limitation of this method is producing blur when the region to be inpainted is thicker than ten pixels.

Zhang et al (2011) introduced fractional-order image inpainting (a projection interpolation method) into metal artifacts reduction in Computer Tomography (CT) images. They introduce a fast non-iterative method based on FMM and coherence transport for metal artifacts reduction (MAR) in computer tomography (CT). Zhang et al (2012) proposed an image inpainting by combining the TV model with fractional derivative called fractional-order TV image inpainting model. They introduced a new class of fractional-order variational image inpainting models in both space and wavelet domains.

All PDE-based methods require difficult implementation process and non-trivial iterative numerical methods such as anisotropic diffusion and multiresolution schemes. Some steps are numerically unstable and inpainting process is slow. For large damaged regions, the results show blocky effect.

\subsection{Convolution-filter-based algorithms}

Oliveira has proposed a fast image inpainting (Olivera et al 2001) method which depends on the convolution operation. The algorithm uses a weighted average kernel that has a zero weight at the centre of the kernel. The algorithm is fast and works well for images which do not have many contrast edges or high frequency components (e.g., natural texture), but produces blurring. In Hadhoud et al (2005), Hadhoud presents a modification of Olivera algorithm permitting implementation time reduction.

Noori et al (2010) have proposed a method in which mask coefficients are calculated using the gradient of the image to be inpainted. The gradient of known pixels in the neighbourhood of a missing pixel is used to compute weights in convolution mask. In Noori et al (2011), inpainting is carried out by convolving the damaged image with a space variant kernel. For every pixel, the kernels are calculated using its neighbours in both space and range domains to implement bilateral filtering. Since the bilateral filters are efficient in denoising, and hence used to inpaint by estimating the lost (damaged) pixel value by its neighbours.

In general, all convolution-based algorithms provide good results only when damaged regions are thin and image does not have many high contrast edges or high frequency components; however, they are faster.

\subsection{Other algorithms}

Roth \& Black (2005) have developed a framework for learning a generic and expressive image priors that capture the statistics of natural scenes which can be used for a variety of machine vision tasks. The approach extends traditional Markov Random Field (MRF) models by learning potential functions over extended pixel neighbourhoods.

Bianjing Bai et al (2011) have proposed completion of missing parts by structure propagation and synthesizing the regions along the salient structures specified by user. After structure completion, a finer algorithm is used to fill in the remaining unknown regions. It can prevent from erroneous matching blocks and reduce the breaking of salient structures.

Bertalmio et al (2003) have proposed to decompose original image into structural and textural sub-images. The structural sub-image is reconstructed by a structure algorithm and the textural sub-image is restored by a texture synthesis approach. Then, two processed 
components are combined to obtain inpainted image. Similar approach is proposed in Grssauer (2004).

Hirani \& Totsuka (1996), Efros \& Leung (1999), Criminisi et al (2004) and Cheng et al (2005) proposed texture inpainting. In these methods, the statistics of damaged regions are matched to statistics of known neighbourhood regions and inpainting is done by either patching the textures at damaged regions or synthesizing the region.

\section{Proposed technique}

The proposed technique is introduced with reference to Bertalmio et al (2000).

The input to the algorithm (Bertalmio et al 2000) is the image to be restored and a mask that delimits the region to be inpainted. As a preprocessing step, the whole original image undergoes anisotropic diffusion smoothing to minimize the influence of noise on the estimation of the direction of the isophotes arriving perpendicular at $\partial \Omega$ (inpainting region boundary). After this, the image enters the inpainting loop, where only value inside $\Omega$ is modified (refer to figure 1).

\subsection{Bertalmio's algorithm}

Consider an image $I$ of size $M \times N$. Let $(i, j)$ be the pixel location inside the inpainting region $\Omega$ and $\Omega$ is the area to be inpainted.

Compute 2D smoothness estimation Lusing Laplacian equation (1)

$$
L^{n}(i, j)=I_{x x}^{n}(i, j)+I_{y y}^{n}(i, j),
$$

where $I_{x x}(i, j) \& I_{y y}(i, j)$ are second-order derivatives of image at pixel $(i, j): 1 \leq i \leq$ $M, 1 \leq j \leq N$ along $x$ axis and $y$ axis, respectively.

The change of $L$ along the direction $\vec{N}$ is given by

$$
\beta^{n}(i, j)=\overrightarrow{\delta L^{n}}(i, j) \cdot \frac{\vec{N}(i, j)}{|\vec{N}(i, j)|},
$$

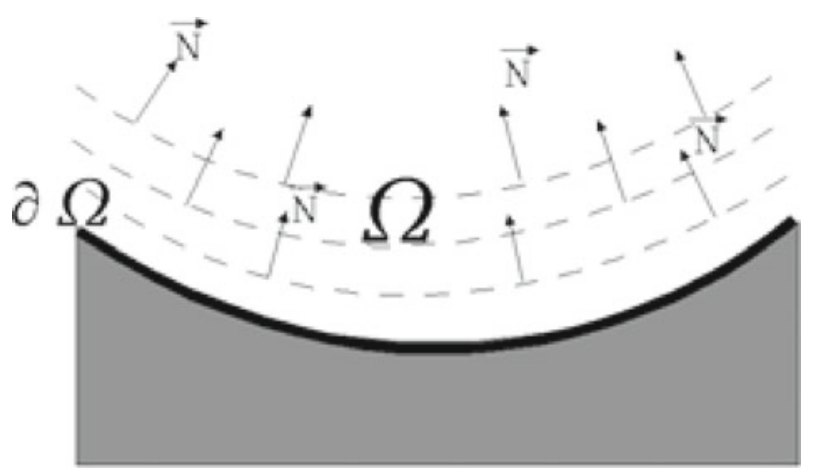

Figure 1. Propagation direction as the normal to the signed distance to the boundary of the region to be inpainted. 
where

$$
\overrightarrow{\delta L^{n}}(i, j)=L^{n}(i+1, j)-L^{n}(i-1, j), L^{n}(i, j+1)-L^{n}(i, j-1)
$$

and the isophote direction $\vec{N} /|\vec{N}|$ is given by

$$
\frac{\vec{N}(i, j)}{|\vec{N}(i, j)|}=\frac{\left(-I_{y}^{n}(i, j), I_{x}^{n}(i, j)\right)}{\sqrt{\left(I_{x}^{n}(i, j)\right)^{2}+\left(I_{y}^{n}(i, j)\right)^{2}}}
$$

Let $I^{n}(i, j)$ stand for each one of the image pixels inside the region $\Omega$ at the inpainting 'time' $n$. Then, the inpainting equation with $\Delta t$ as improvement factor is given by

$$
I^{n+1}(i, j)=I^{n}(i, j)+\Delta t \beta^{n}\left|\Delta I^{n}(i, j)\right| \forall(i, j) \in \Omega,
$$

where $\left|\Delta I^{n}(i, j)\right|$ is slope-limited version of the norm of the gradient which is added in order to provide stability. The slope-limited norm ensures that extremely large values will not be obtained for $I^{n}(i, j)$ and keeps the resulting image from 'blowing up'. The slope-limited norm is given by

$$
\left|\nabla I^{n}(i, j)\right|=\left\{\begin{array}{l}
\sqrt{\left(I_{x b m}^{n}(i, j)\right)^{2}+\left(I_{x f M}^{n}(i, j)\right)^{2}+\left(I_{y b m}^{n}(i, j)\right)^{2}+\left(I_{y f M}^{n}(i, j)\right)^{2}} \\
\text { when } \beta^{\mathrm{n}}>0 \\
\sqrt{\left(I_{x b M}^{n}(i, j)\right)^{2}+\left(I_{x f m}^{n}(i, j)\right)^{2}+\left(I_{y b M}^{n}(i, j)\right)^{2}+\left(I_{y f m}^{n}(i, j)\right)^{2}} \\
\text { when } \beta^{\mathrm{n}}<0
\end{array}\right.
$$

Note that the inpainting algorithm terminates on arriving steady state.

3.1a Limitations of the technique: (i) More number of iterations for larger inpainting area are needed, since the isophotes are of small size. (ii) It is also apparent from equation (3), the isophote lines may cross one another, hence inpainting loop has to be interleaved by anisotropic diffusion. More number of iterations, lead to blurring and result in inpainting error. (iii) For larger inpainting areas due to step (2), artifacts are generated. (iv) The algorithm is inherently numerically unstable, as it involves normalization of gradient and Laplacian.

\subsection{Proposed algorithm}

The limitations of technique of Bertalmio et al (2000) are addressed by modifying the inpainting equation (5) as equation (7) and the inpainted image pixel is given by

$$
I(i, j)=\operatorname{median}(I(x, y)) \quad \text { for } \forall(i, j) \in \Omega,
$$

where $x=i-D / 2: i+D / 2$ and $y=j-D / 2: j+D / 2$.

Here, inpainting is interpreted as median filtering, a nonlinear order statistics filtering. According to Ioannis \& Anastasios (1992), the median is the maximum likelihood estimate (MLE) 
of location for the Laplacian distribution (Appendix A). Hence, using (7) over (5) gives better estimate of the pixel $I(i, j) \in \Omega$.

The parameter $D$ is chosen almost same as the width of inpainting area and is an even number. This determines the influence of pixel exterior to the inpainting area (refer to figure 2). The median separates higher half of a samples to the lower half in a probability distribution function, satisfying the criteria

$$
P(x \leq m) \geq 0.5 \text { and } P(x \geq m) \geq 0.5,
$$

where $m$ is the median value of the probability distribution function $P(x)$. Hence, the deviation of the neighbours from the median value is never greater than 0.5 , i.e., the probability of

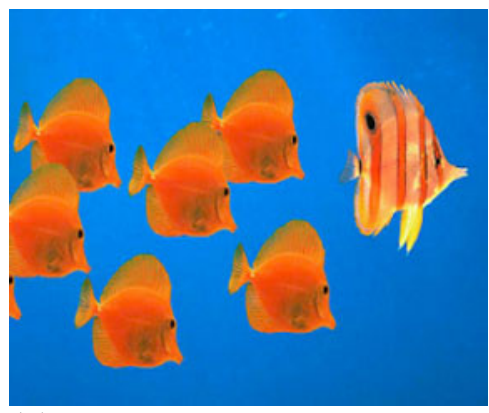

(a)

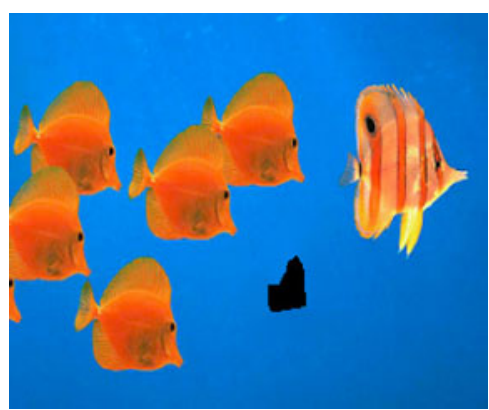

(c)

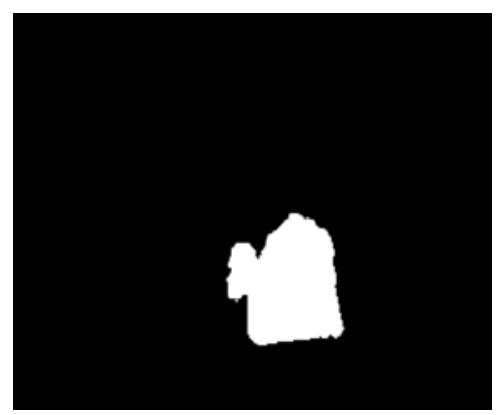

(b)

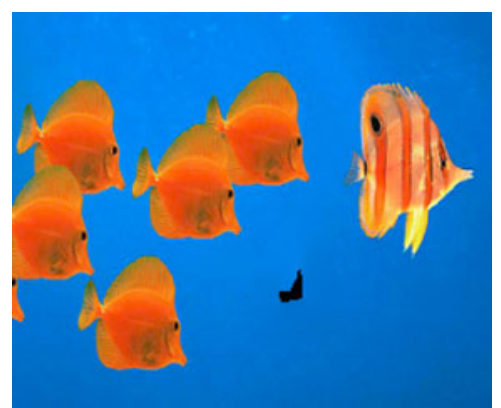

(d)

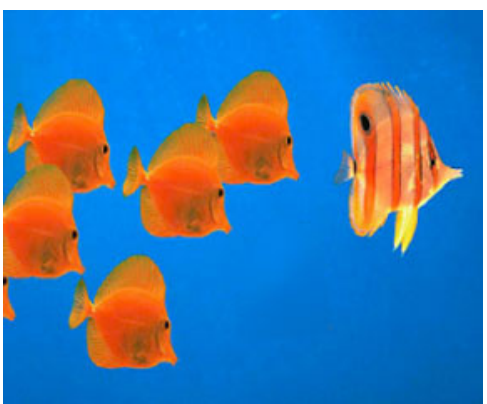

(e)

Figure 2. (a) Original image. (b) Mask image. (c) Inpainted image with $D=30$ in which large inpainting area is left. (d) Inpainted image with $D=35$ in which inpainting area is reduced. (e) Inpainted image with $D=42$ in which inpainting area is completely filled by surrounding information. 
inpainted pixel is always greater than 0.5. This understanding is important in the context of the current study because it clearly indicates that if median propagation is adopted for the inpainting process then the inpainted pixel will have probability greater than or equal to 0.5 . It is important to have larger range of values across the inpainting area to estimate the pixel distribution correctly.

\subsection{Detailed algorithm}

Consider an original image $I(i, j): 1 \leq i \leq M, 1 \leq j \leq N$ and is an input to our algorithm. At the end of the algorithm, the inpainted image replaces input image.

\section{Start}

Select the parameter $D$ which is proportional to the width of inpainting area.

For every pixel $(i, j)$ belonging to the inpainting area $\Omega$

Select Range $=((i-D / 2: i+D / 2),(j-D / 2: j+D / 2))$

$R=I$ (Range, red), $G=I$ (Range, green), $B=I($ Range, blue)

For grey level images, any one component can be used or may be suitably modified.

Apply median filtering:

Take median of $\mathrm{R}, \mathrm{G}$ and $\mathrm{B}$ component independently i.e.,

$R=\operatorname{median}(R), G=\operatorname{median}(G), B=\operatorname{median}(B)$

Diffuse $(i, j)$ pixel of $I$ with $[\mathrm{R}, \mathrm{G}, \mathrm{B}]$

$I(i, j)=[R, G, B]$

End.

Repeat above steps for all pixels $(i, j) \in$ to the inpainting area $\Omega$.

\subsection{An example of median filtering}

Consider $D=2$ for figure 3 a of inpainting area $3 * 3$ and $\mathrm{P}_{5}$ is located at $(i, j)$, then $\mathrm{P}_{5}$ is replaced by the median value of $\mathrm{P}_{1}, \mathrm{P}_{2}, \mathrm{P}_{3}, \mathrm{P}_{4}, \mathrm{P}_{5}, \mathrm{P}_{6}, \mathrm{P}_{7}, \mathrm{P}_{8}$, and $\mathrm{P}_{9}$, while doing so the algorithm checks

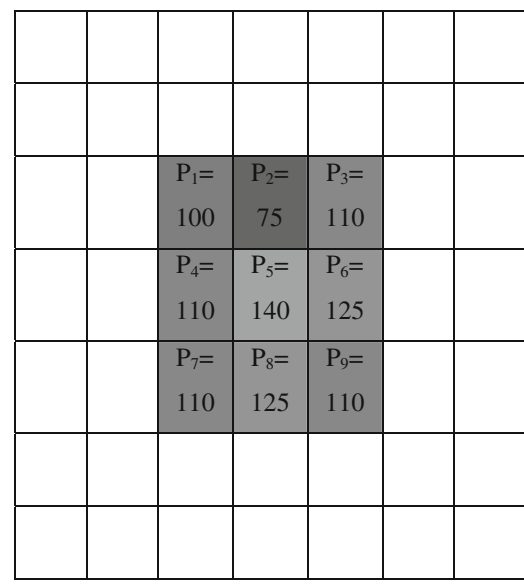

(a)

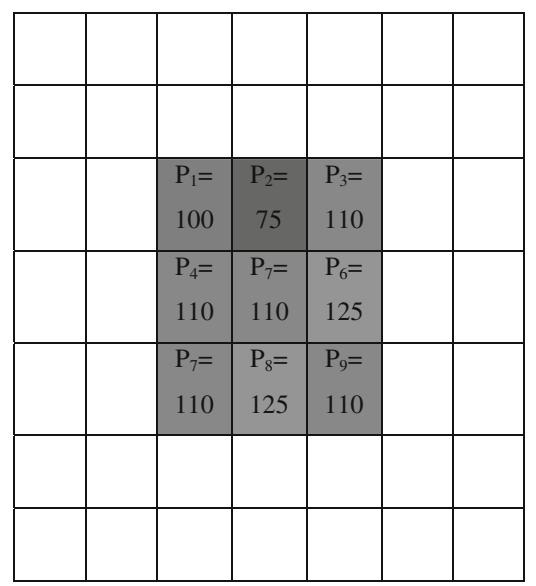

(b)

Figure 3. (a) Original image. (b) Inpainted image. 
Table 1. A typical illustration of median diffusion for an image patch of $3 \times 3$.

\begin{tabular}{|c|c|c|c|}
\hline$P_{1}=100$ & $\mathrm{P}_{2}=75$ & $\mathrm{P}_{2}=75$ & $\mathrm{P}_{2}=75$ \\
\hline$P_{2}=75$ & $P_{5}=100$ & $\mathrm{P}_{5}=100$ & $P_{5}=100$ \\
\hline $\mathrm{P}_{3}=110$ & $\mathrm{P}_{3}=110$ & $\mathrm{P}_{3}=110$ & $\mathrm{P}_{3}=110$ \\
\hline $\mathrm{P}_{4}=110$ & $\mathrm{P}_{4}=110$ & $\mathrm{P}_{4}=110$ & $\mathrm{P}_{4}=110$ \\
\hline$P_{5}=140$ & $\mathrm{P}_{6}=125$ & $P_{7}=110$ & $\mathrm{P}_{7}=110$ \\
\hline$P_{6}=125$ & $\mathrm{P}_{7}=110$ & $\mathrm{P}_{6}=125$ & $\mathrm{P}_{9}=110$ \\
\hline $\mathrm{P}_{7}=110$ & $\mathrm{P}_{8}=125$ & $\mathrm{P}_{9}=110$ & $\mathrm{P}_{6}=125$ \\
\hline $\mathrm{P}_{8}=125$ & $\mathrm{P}_{9}=110$ & $\mathrm{P}_{8}=125$ & $\mathrm{P}_{8}=125$ \\
\hline$P_{9}=110$ & $P_{5}=140$ & $\mathrm{P}_{5}=140$ & $\mathrm{P}_{5}=140$ \\
\hline
\end{tabular}

the gradient and performs anisotropic diffusion as shown in table 1. Hence, there is no need for interleaving anisotropic diffusion.

\section{Results}

The proposed algorithm is tested on a variety of images to investigate the performance of inpainting. More than 100 images are used to inpaint. In this section, only few samples are quoted. The images have homogenous or heterogeneous background. In homogenous image regions, noise standard deviation is approximately equal to signal distribution. We found out that the signal deviation is about $15 \%$ in our test images with homogenous background (figures 4, 5 and 6).

The test set constitutes of:

- Standard images used by image processing community: Lena, cameraman, fruits.

- Synthetic images using available software: checker board pattern, computer LAN.

- Natural images taken by the authors or collected by the authors: Group photograph, Tajmahal, Ganguly with bat, group of children, archeological images.

The original images are clean images, and these images are artificially degraded by drawing random lines on them. The random lines are treated as scratches or areas of inpainting. These degraded images are then inpainted. The comparison of proposed algorithm is carried out by using the codes written by Bertalmio et al (2000, Image Inpainting) and Stefan Roth (2005, FoE) without any major changes. The proposed algorithm is implemented in MATLAB R2007b.

Evaluation of inpainting by perceptual quality is subjective in nature and hence a quantitative assessment is more objective to compare various inpainting algorithms. However, this is a difficult task and there is no common method for evaluating inpainting algorithms.

In our study, the quantitative evaluation is performed by calculating peak signal to noise ratio (PSNR) and is defined as

$$
\operatorname{PSNR}=20 \log _{10}\left(\frac{255}{\operatorname{RMSE}(u, v)}\right), \quad \text { where } \operatorname{RMSE}=\sqrt{\frac{\sum_{i, j}\left(u_{i, j}-v_{i, j}\right)^{2}}{M N}}
$$

and $u$ is original image, $v$ is inpainted image and $M \times N$ is size of $\mathrm{u}$ and $\mathrm{v}$. This measure is only well-suited for the testing purpose, as original image is damaged and then restored. In practical 


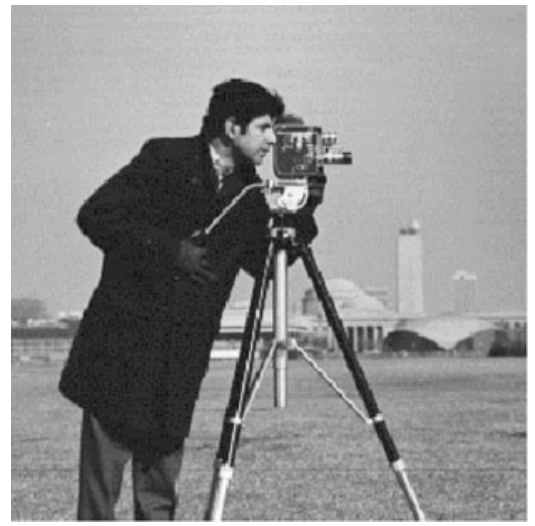

(a)

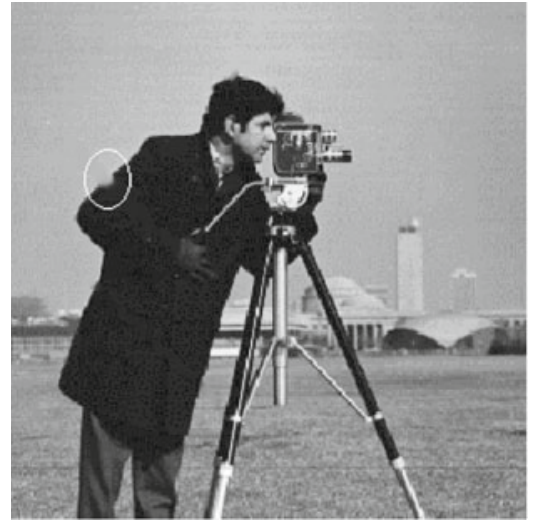

(c)

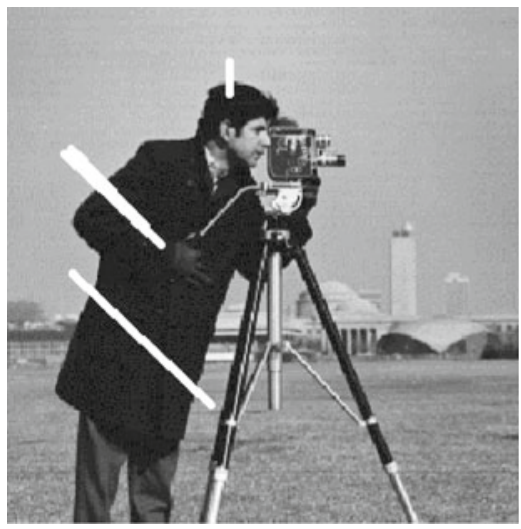

(b)

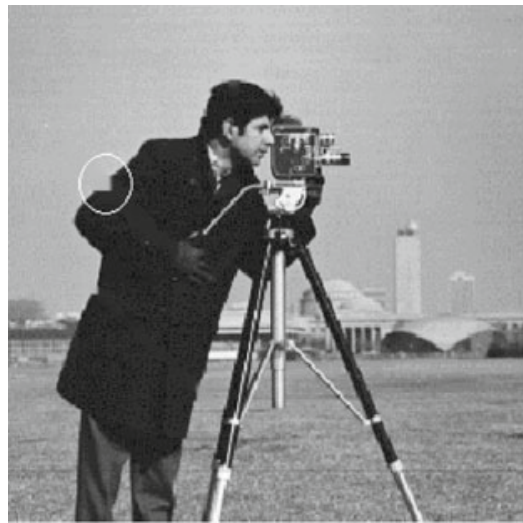

(d)

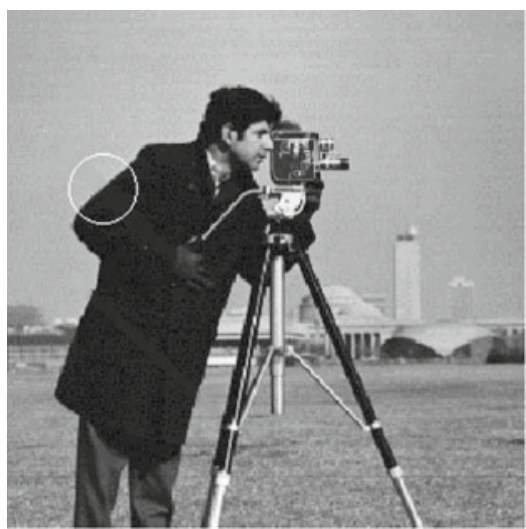

(e)

Figure 4. (a) Original cameraman image. (b) Damaged image. (c) Inpainted image by Bertalmio algorithm with PSNR $39.6162 \mathrm{~dB}$. (d) Inpainted image by FoE algorithm with PSNR $37.2435 \mathrm{~dB}$. (e) Inpainted image by our algorithm with PSNR $40.7218 \mathrm{~dB}$. Note the encircled portion of the image, figure $4 \mathrm{e}$ and is the crucial area of inpainted region. The figure 4e shows natural edge construction and visually pleasing inpainting obtained by our algorithm. 


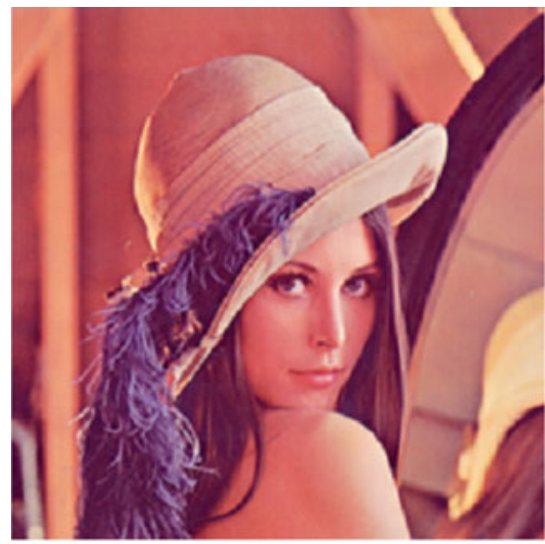

(a)

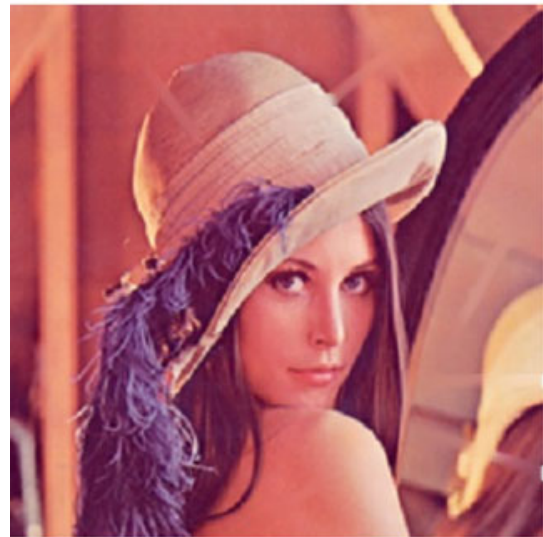

(c)

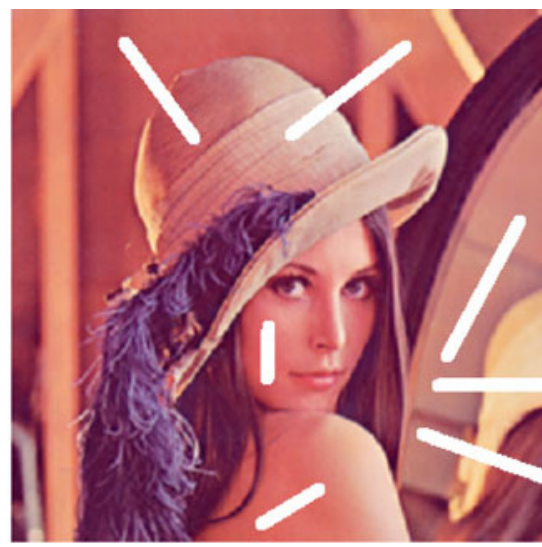

(b)

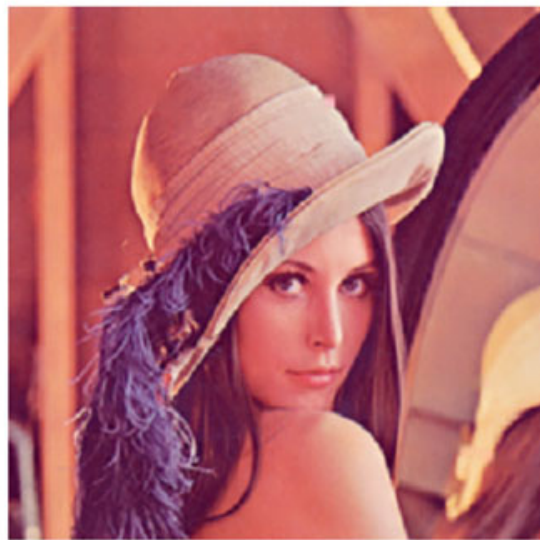

(d)

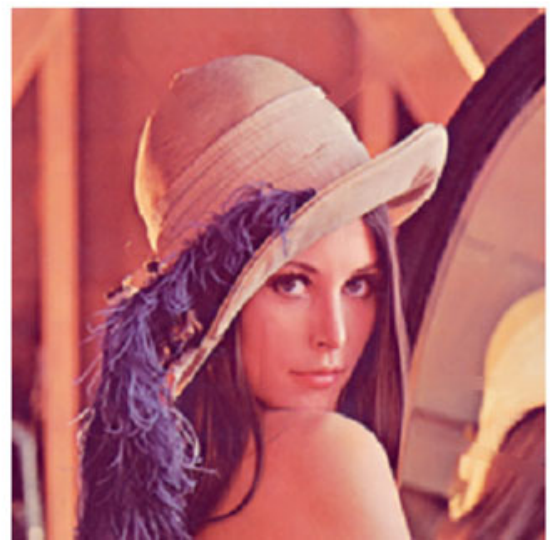

(e)

Figure 5. (a) Original lena image. (b) Damaged image. (c) Inpainted image by Bertalmio with PSNR $38.3164 \mathrm{~dB}$. (d) Inpainted image by FoE algorithm algorithm with PSNR $41.9601 \mathrm{~dB}$. (e) Inpainted image by proposed algorithm with PSNR $44.5642 \mathrm{~dB}$. 


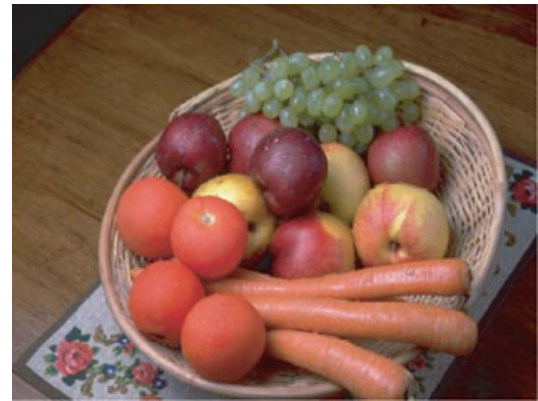

(a)

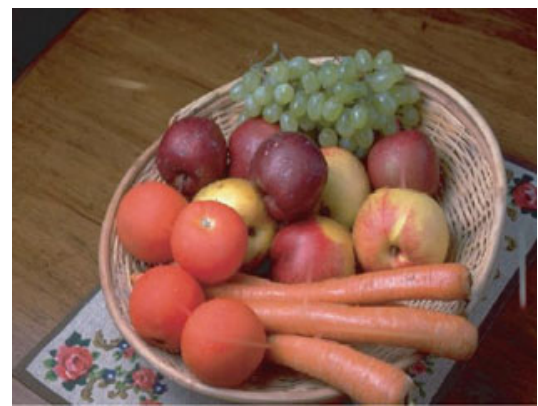

(c)

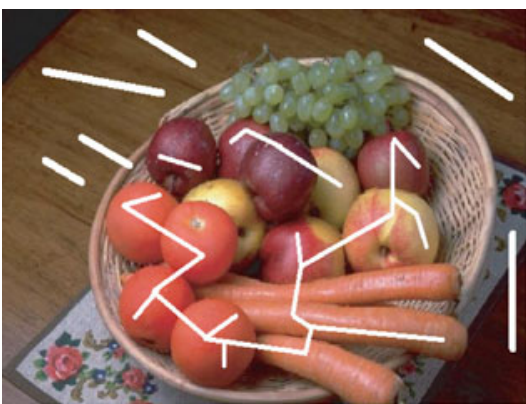

(b)

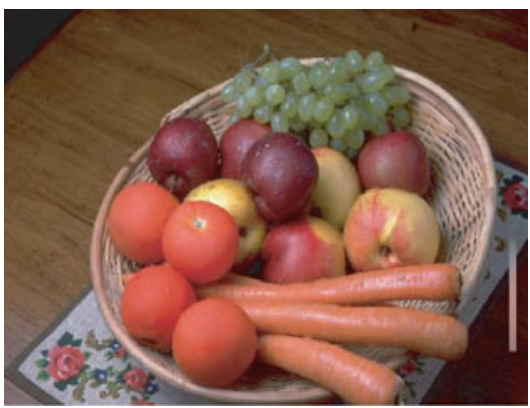

(d)

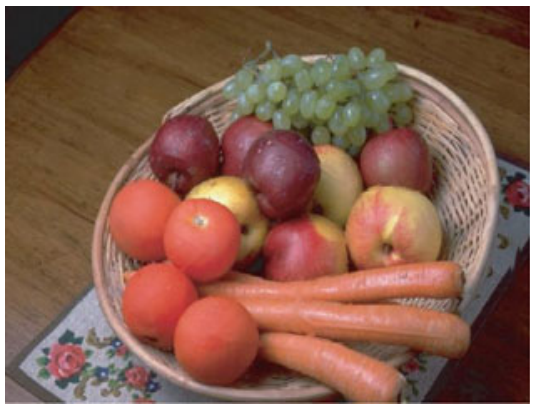

(e)

Figure 6. (a) Original image of fruits and vegetables. (b) Original image is damaged by putting white lines. (c) Inpainted image by Bertalmio algorithm with PSNR $34.6656 \mathrm{~dB}$. (d) Inpainted image by FoE algorithm PSNR 31.5572 dB. (e) Inpainted image by our algorithm with PSNR 42.1953 dB.

Table 2. Comparison of our algorithm with Bertalmio and FoE algorithm. Both Bertalmio and FoE results are obtained after 2000 iterations.

\begin{tabular}{lcccc}
\hline $\begin{array}{l}\text { Figure } \\
\text { No. }\end{array}$ & Image & $\begin{array}{c}\text { PSNR of Bertalmio's } \\
\text { algorithm }(\mathrm{dB})\end{array}$ & $\begin{array}{c}\text { PSNR of FoE } \\
\text { algorithm }(\mathrm{dB})\end{array}$ & $\begin{array}{c}\text { PSNR of proposed } \\
\text { algorithm }(\mathrm{dB})\end{array}$ \\
\hline 4 & Cameramen & 39.6162 & 37.2435 & 40.7218 \\
5 & Lena & 38.3164 & 41.9601 & 44.5642 \\
6 & Fruits \& Vegetables & 34.6656 & 31.5572 & 42.1953 \\
7 & Synthetic & 34.6656 & 23.1986 & 51.1575 \\
8 & Synthetic & 32 & 23.9839 & 43.9511 \\
9 & Group photograph & 36.0144 & 33.4102 & 40.1383 \\
\hline
\end{tabular}




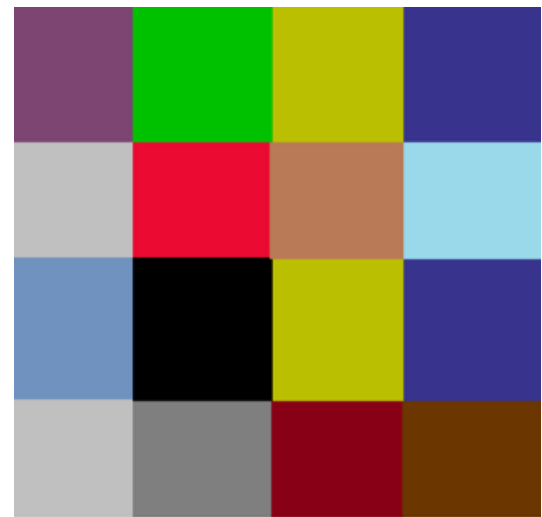

(a)

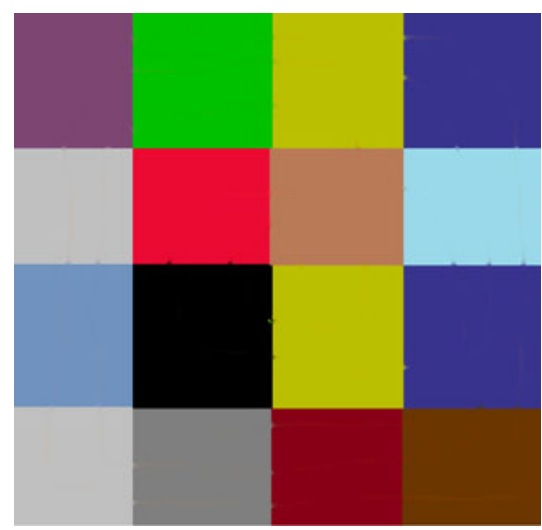

(c)

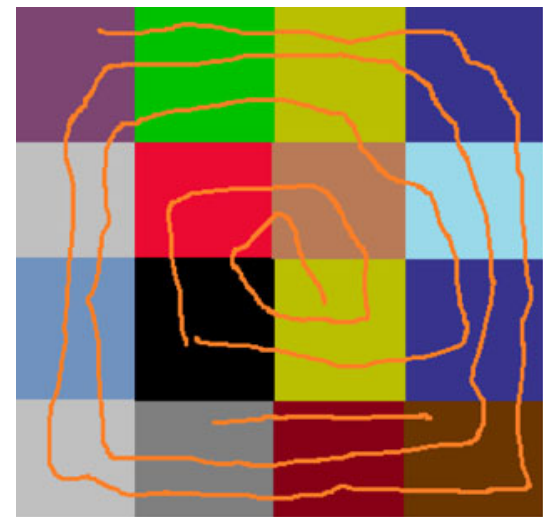

(b)

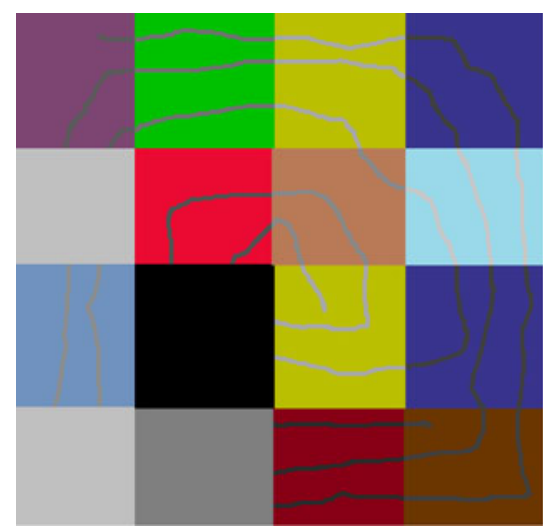

(d)

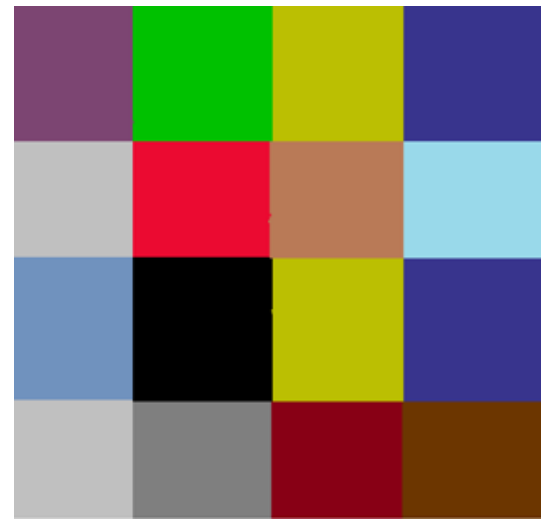

(e)

Figure 7. (a) Original synthetic image. (b) Damaged image. (c) Inpainted image by Bertalmio algorithm with PSNR 34.6656 dB. (d) Inpainted image by FoE algorithm with PSNR 23.1986 dB. (e) Inpainted image with proposed algorithm with PSNR 51.1575 dB. 
applications, such a measure is not feasible, because there is no original image. Hence, we have to rely on qualitative evaluation.

\subsection{Standard images with heterogeneous background}

Initially, the algorithm is tested on standard images viz. Cameraman, Lena and Fruits. These images are the most commonly used by image processing community and provide benchmark results for the efficacy of any image processing algorithms. The PSNR is calculated for each inpainted image and tabulated in table 2.

\subsection{Synthetic images}

Synthetic image is created using paint software. The idea to use synthetic image is to extend the inpainting algorithm to reconstruct the edges at the critical portions of the images. The concept can be readily seen from the synthetic image and the scratches or patches put on the original

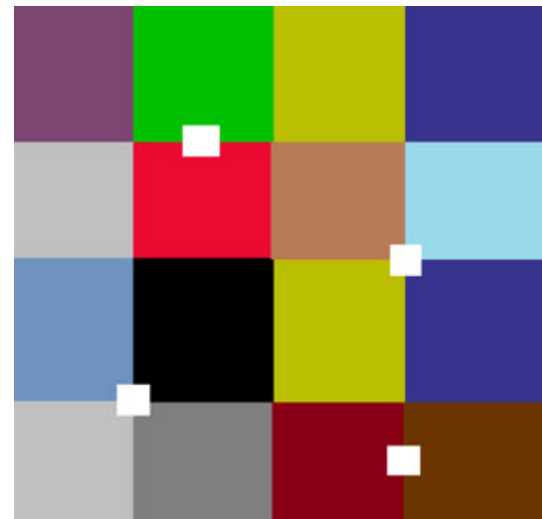

(a)

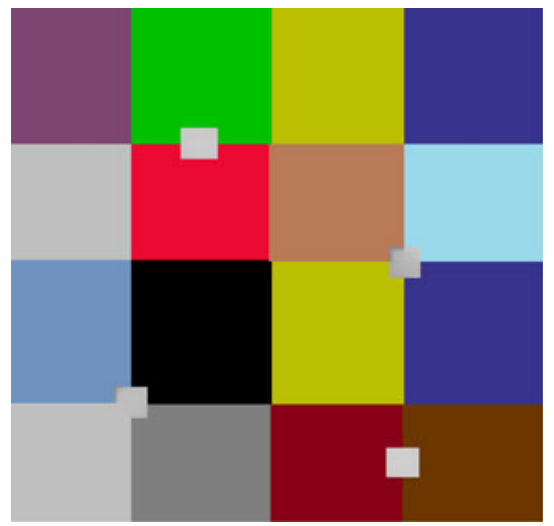

(c)

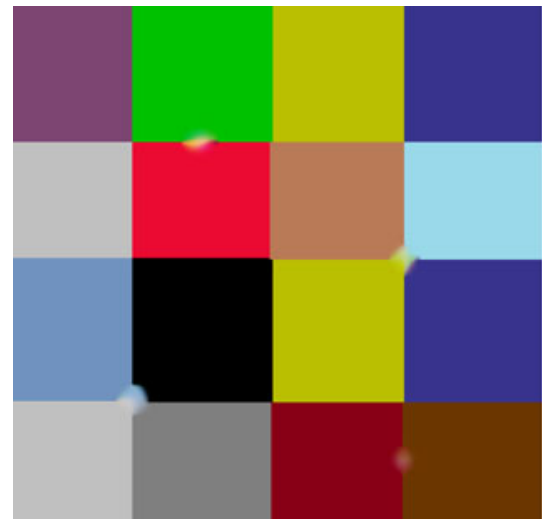

(b)

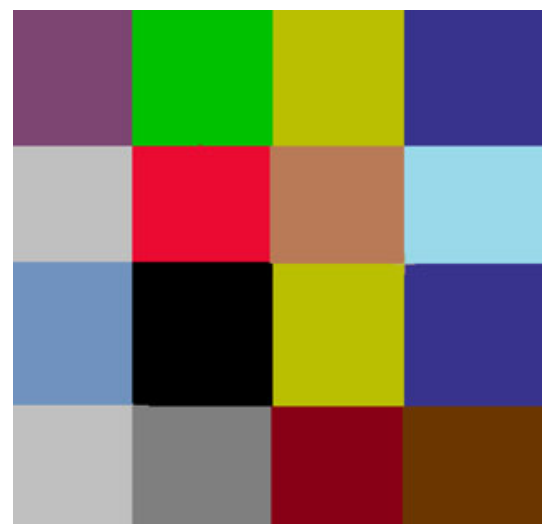

(d)

Figure 8. (a) Original image (figure 7a) is damaged by putting white patches. (b) Inpainted image by Bertalmio algorithm with PSNR $32 \mathrm{~dB}$. (c) Inpainted image by FoE algorithm algorithm with PSNR $23.9839 \mathrm{~dB}$. (d) Inpainted image by proposed algorithm with PSNR $43.9511 \mathrm{~dB}$. 


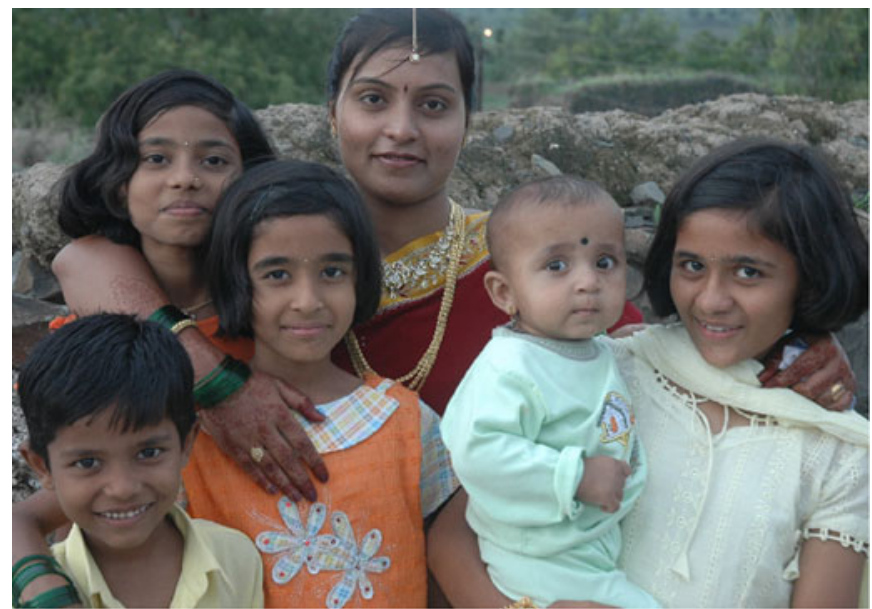

(a)

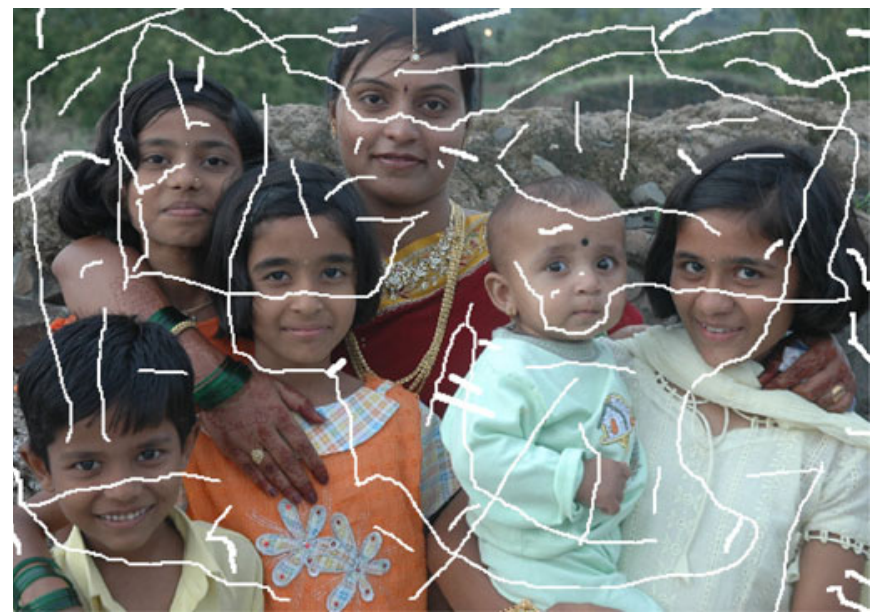

(b)

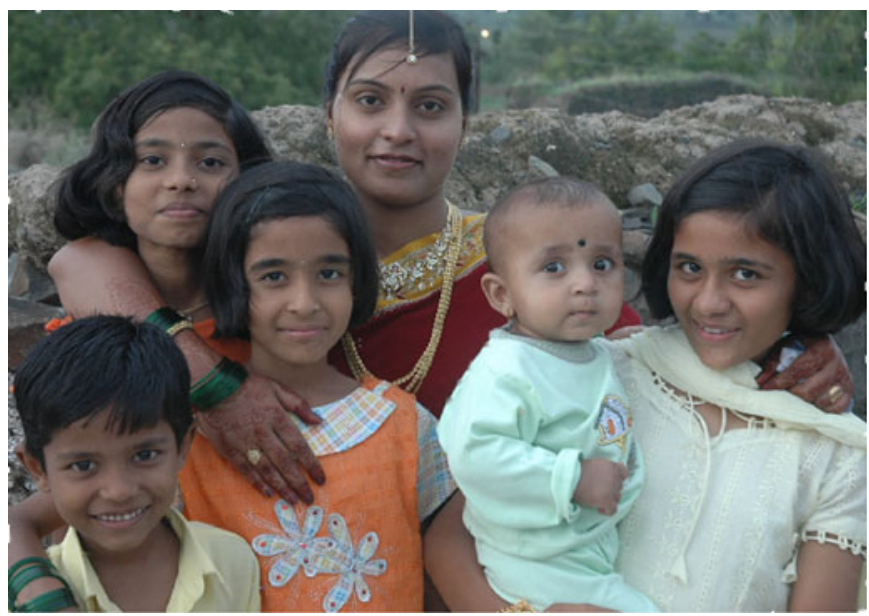

(c) 
image and restored. The PSNR is calculated for each method and is tabulated in table 2. Note that the inpainting is difficult for the other algorithms as they do not address edge preservation. Since algorithm is based on diffusion Bertalmio et al, it inpaints homogeneous regions but fails when two or more homogeneous regions are adjacent (figure 8b). The FoE technique is based on MRF learning and filtering, the edge information is lost in the inpainting processes and residues appear in the results (figures $7 \mathrm{~d}$ and $8 \mathrm{c}$ ).

\subsection{Natural image with heterogeneous background}

An important application of inpainting is to restore the colour photographs damaged due to scratches. A sample colour photograph taken in day light with natural background is used.

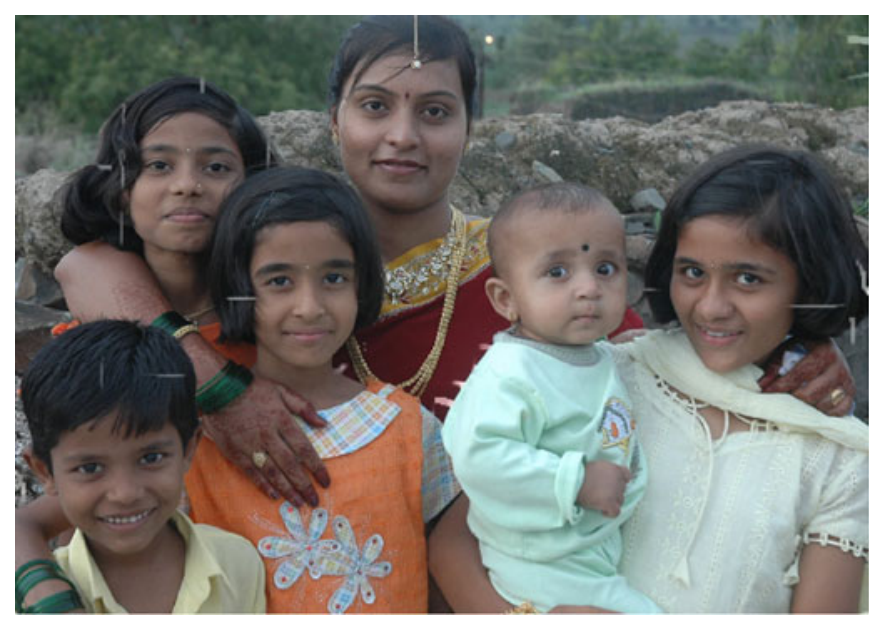

(d)

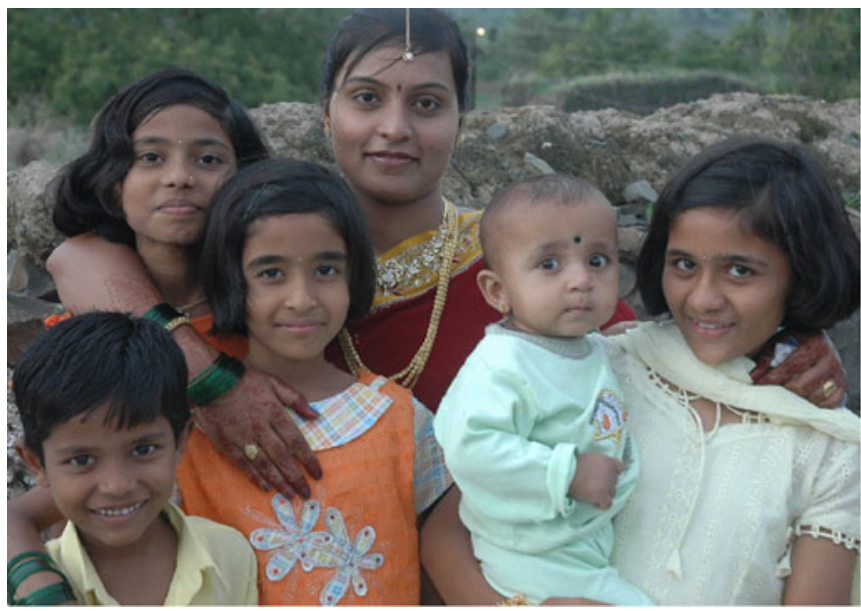

(e)

Figure 9. (a) Original group photograph. (b) Damaged image by putting white random lines. (c) Inpainted image by Bertalmio algorithm with PSNR $36.0144 \mathrm{~dB}$. (d) Inpainted image by FoE algorithm with PSNR $33.4102 \mathrm{~dB}$. (e) Inpainted image by the proposed algorithm with PSNR $40.1383 \mathrm{~dB}$. 


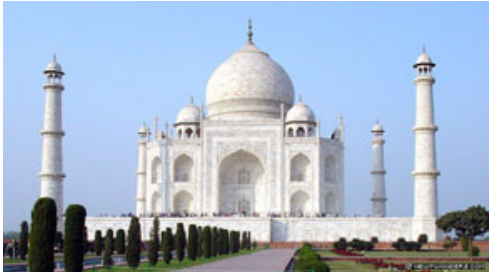

(a)

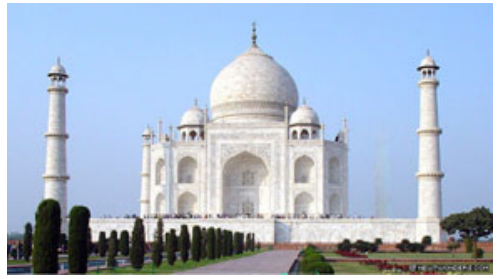

(c)

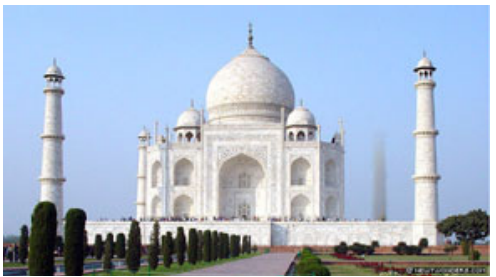

(b)

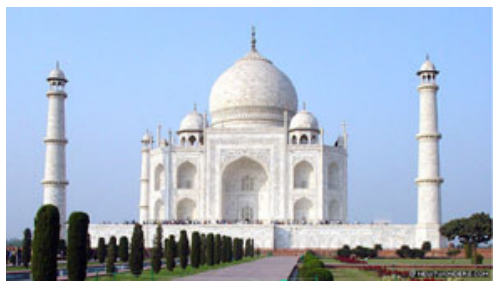

(d)

Figure 10. (a) Original Tajmahal image. (b) Bertalmio result at 1500 iterations. (c) Result of FoE at 1500 iterations. (d) Result of the proposed algorithm.

Figures $9 \mathrm{a}$ and $\mathrm{b}$ show the original photograph and scratched photograph, respectively. Figures $9 \mathrm{c}, \mathrm{d}$ and e show inpainted images. It can be seen that the quality of inpainting obtained by the proposed algorithm is superior to other algorithms. The PSNR is tabulated in table 2 .

\subsection{Object removal}

The PSNR measure and qualitative assessments give identical interpretations. Therefore, we envisage the algorithms to perform similarly and hence can be extended to any practical degraded image expecting identical performance. The algorithm is tested to remove bigger objects from a given image and results of the proposed algorithm are shown in figures $10 \mathrm{~d}, 11 \mathrm{~d}, 12 \mathrm{~d}$ and $13 \mathrm{~d}$. It can be seen from the results that, for larger inpainting areas, Bertalmio et al and FOE techniques fail to inpaint. Further, the FoE technique fails at the border of the image (figure 13c).

\section{Applications}

Image inpainting techniques are in use for various applications like removal of scratches, removal of superimposed data, restoring damaged/missing portions and removal of objects from the images (shown in previous section).

\subsection{Removal of natural scratch}

The first and probably first application of inpainting is to remove the scratches of old portraits and old paintings. Here, we applied the proposed algorithm to an old photograph (figure 14a) with a scratch and restored it (figure 14b). 


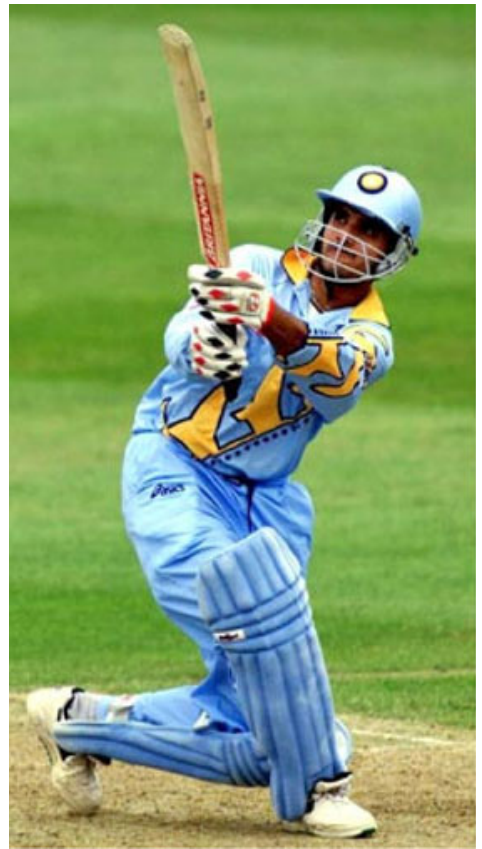

(a)

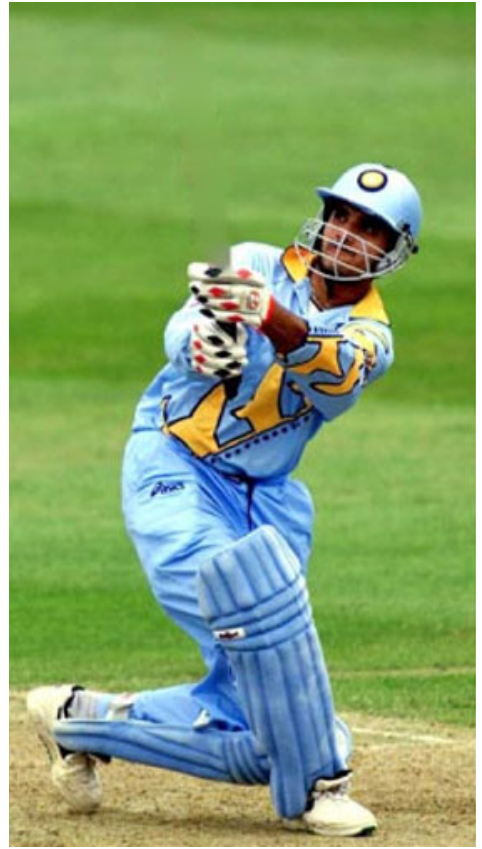

(c)

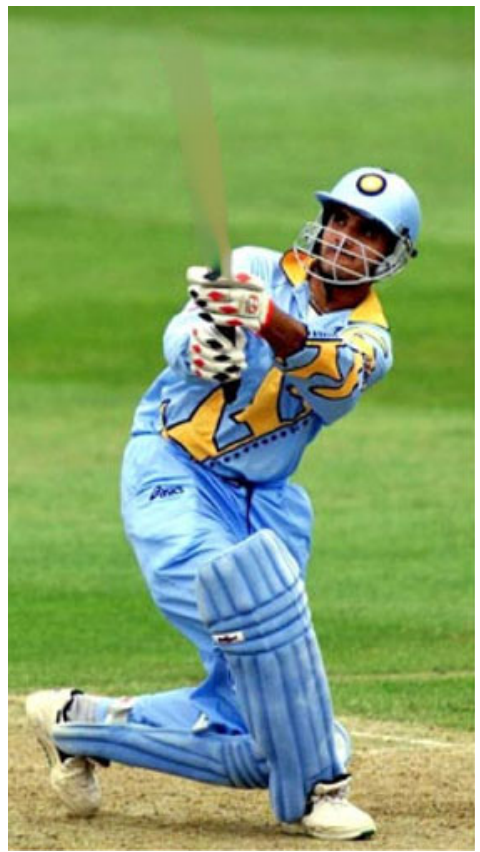

(b)

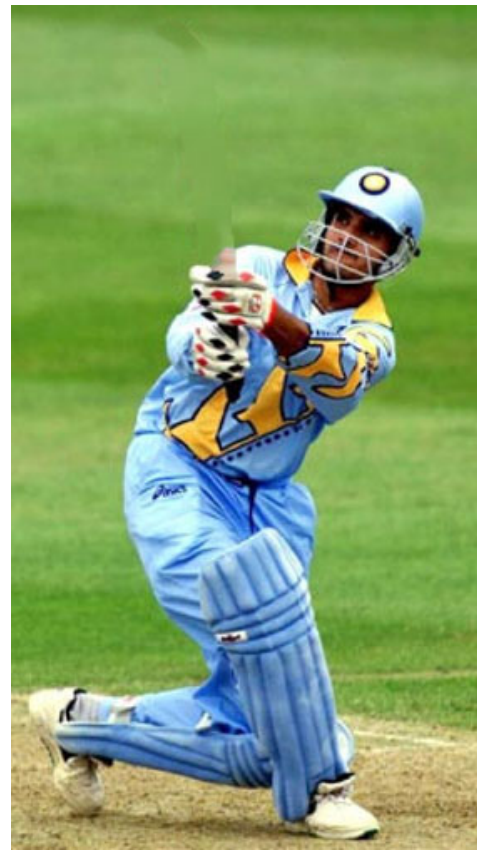

(d)

Figure 11. (a) Batsman Ganguly's original image. (b) Bertalmio with 3000 iterations. (c) FoE with 3000 iterations. (d) Proposed technique result. 


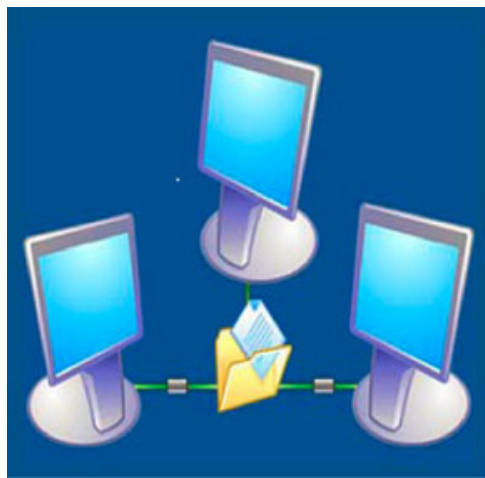

(a)

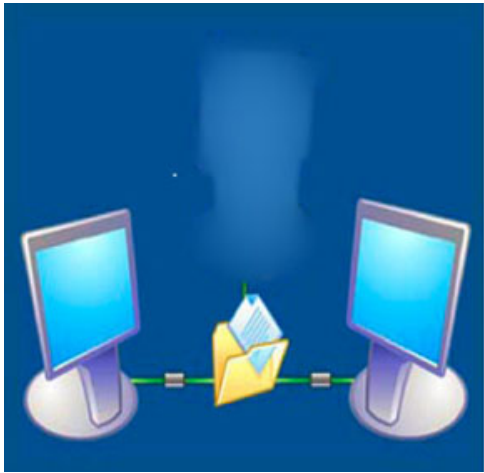

(c)

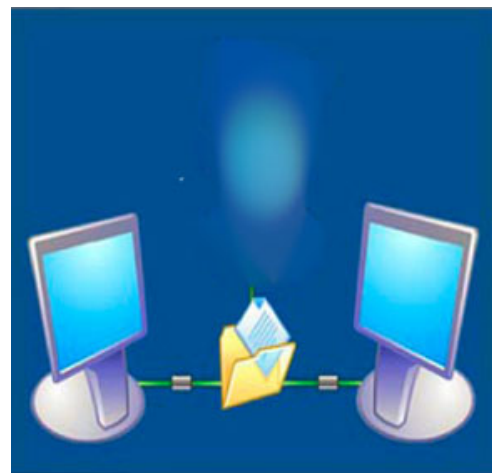

(b)

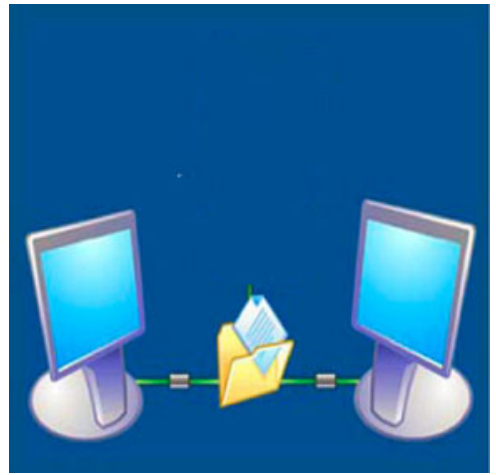

(d)

Figure 12. (a) Low intensity RGB image. (b) Result of Bertalmio at 4000 iterations. (c) Result of FoE at 4000 iterations. (d) Result of the proposed technique.

\subsection{Archaeological application}

We envisage that inpainting may aid archaeological work to reconstruct old monuments. Most of the archaeological monuments have developed cracks and restoration work of the monuments may be helped by providing possible appearance of the monument using inpainting. Figures 15a and 16a show the archaeological images of portions of Kalalingeshwar temple, Kalgi of Gulbarga district, Karnataka, India, taken in daylight. Figures $15 \mathrm{~b}$ and $16 \mathrm{~b}$ show the restored archaeological images, respectively.

\subsection{Removal of superimposed characters on image}

An interesting application of inpainting is to remove the superimposed characters which are in the form of writing. The image hides behind the text and by inpainting the image is extracted. Figure 17a shows image with superimposed text and figure $17 \mathrm{~b}$ shows extracted image by our proposed algorithm.

\subsection{Removal of specular regions in endoscopic image}

Endoscopic images usually have a glossy appearance showing specular highlights due to specular reflection of light source (figure 18a). These image features can negatively affect the 


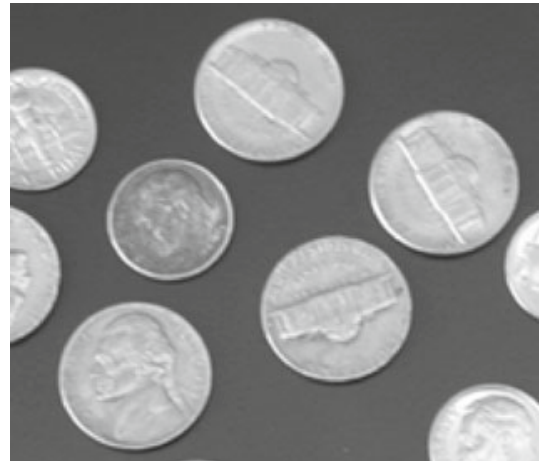

(a)

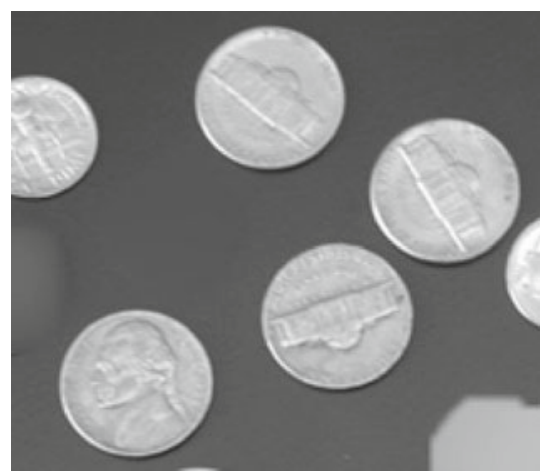

(c)

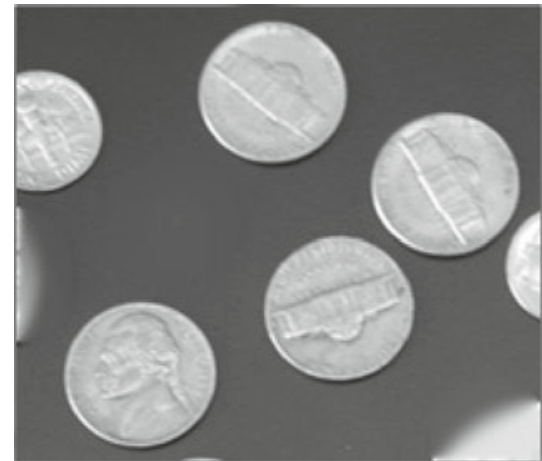

(b)

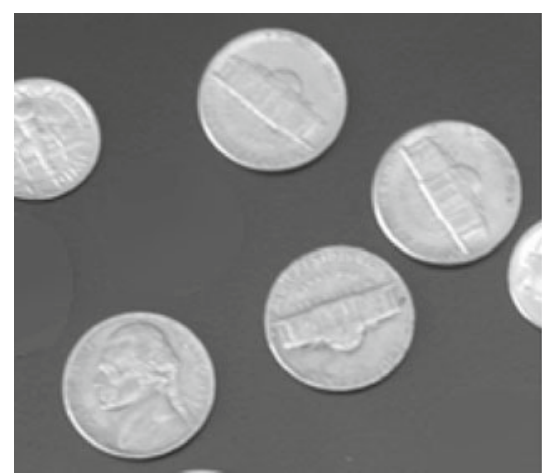

(d)

Figure 13. (a) Original image with multiple objects. (b) Bertalmio at 2000 iterations. (c) FoE at 2000 iterations which fails at the borders. (d) Result of proposed method.

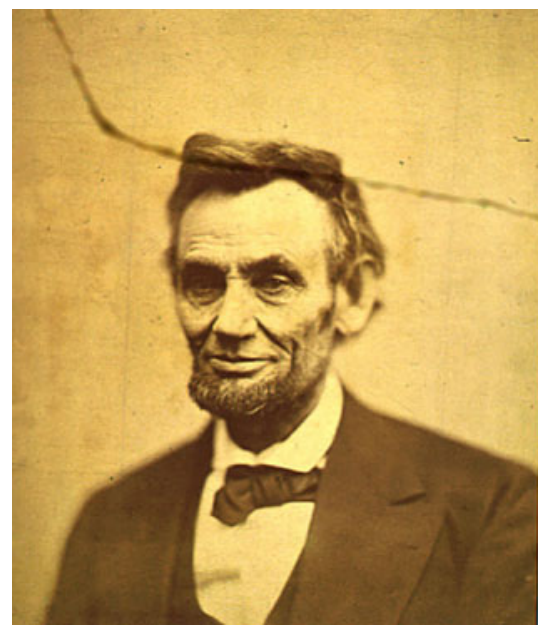

(a)

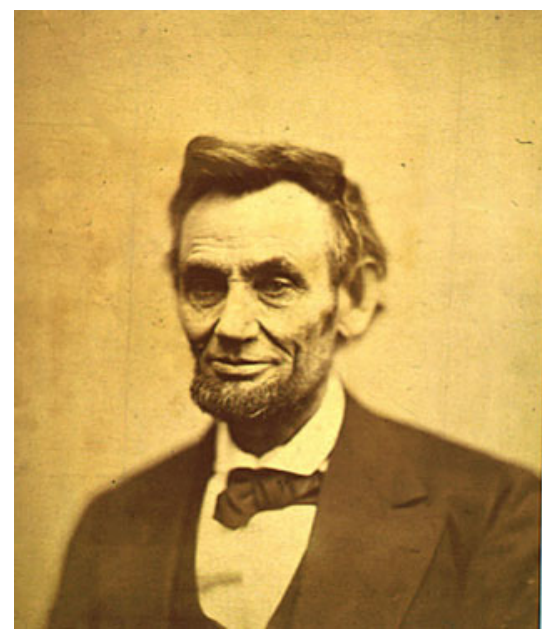

(b)

Figure 14. (a) Actual scratched image of Abraham Lincoln. (b) Restored image by proposed algorithm. 


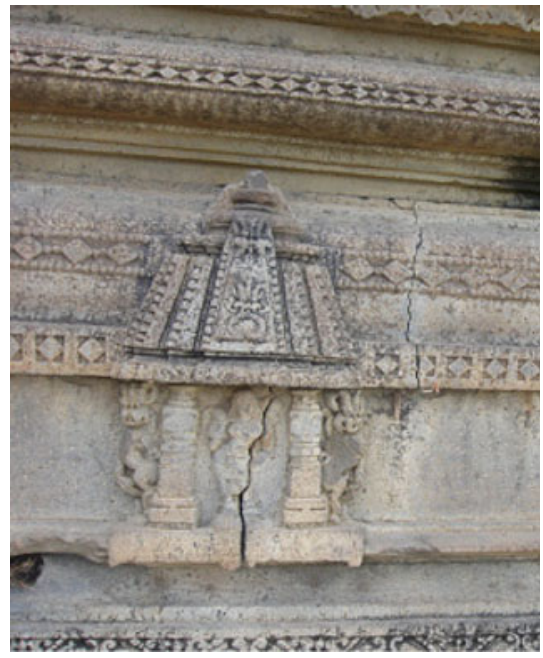

(a)

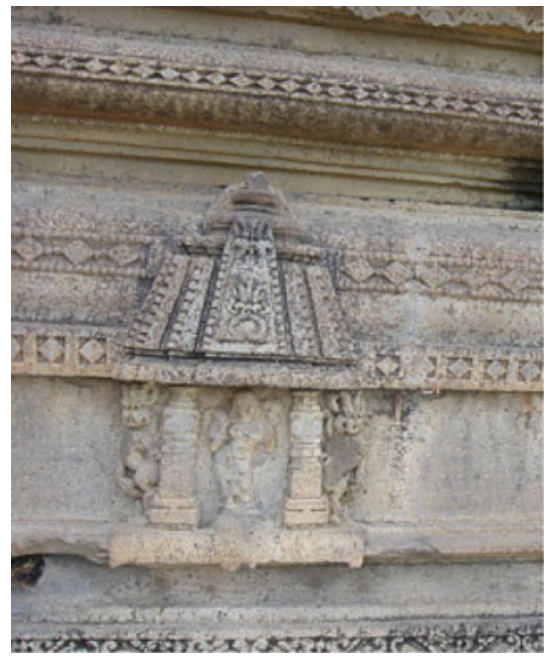

(b)

Figure 15. (a) Original image. (b) Restored image.

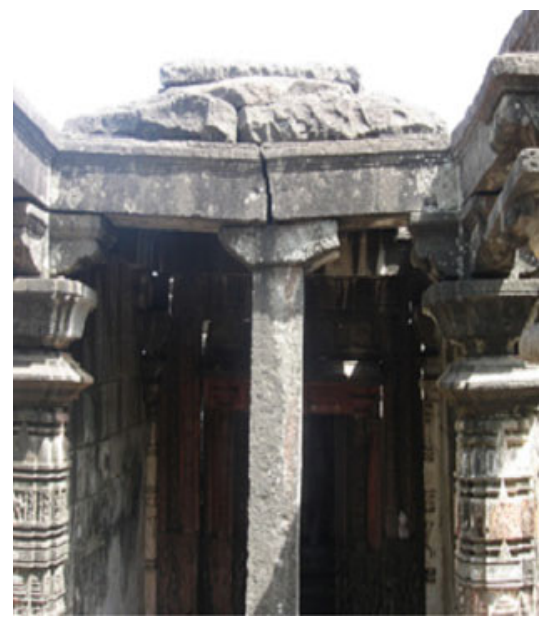

(a)

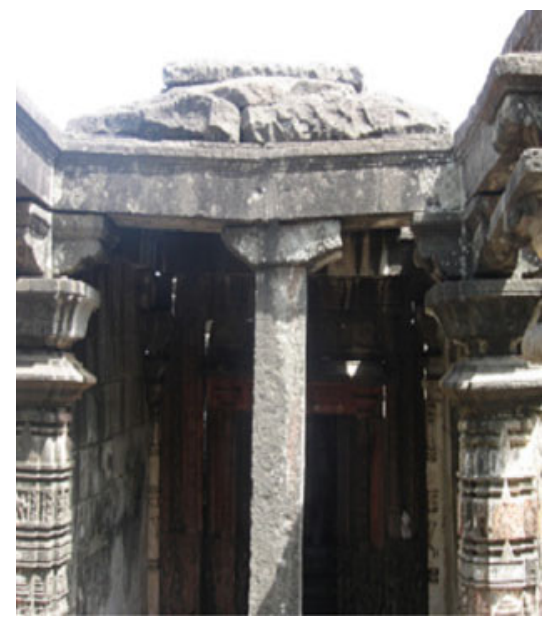

(b)

Figure 16. (a) Original image. (b) Restored image.

perceived image quality. Inpainting method alters the specular regions in a way that eliminates the negative effect of endoscopic image and gives a visually pleasant result as shown in figure $18 \mathrm{~b}$.

\section{Conclusion}

The algorithms proposed by Bertalmio et al and Roth and Black are most widely adopted techniques, but these techniques are well-suited to small inpainting regions. In this study, extensive 
Digital Jmage Inpainting : Techniques, analysis and Applications: Bertalmions. FoE, Proposed Techniqueee lmage inpainting is the technique of filling-in the missing regions and andremoving un wanted objects from an an image by diffusing the pixel information from the neighborhood pixels. Image inpainting tech techniques are in use over a long time for various $\mathrm{s}$ applications like removal of scratches, restoring and damaged $/ \mathrm{missing}$ portions or removal of objects reg from the images etc. In this work, we present a sim simple, yet unexplored (digital) inpainting technique using median filter, one of the most popular popul nonlinear (order statistics) filters. The median is is maximum likelihood estimate (MLE) of location for the Laplacian distribution. Hence, the proposed tech algorithm diffuses median Median is Maximum likeli likelihoo estimate of locaton for Laplacian distirbuton

(a)

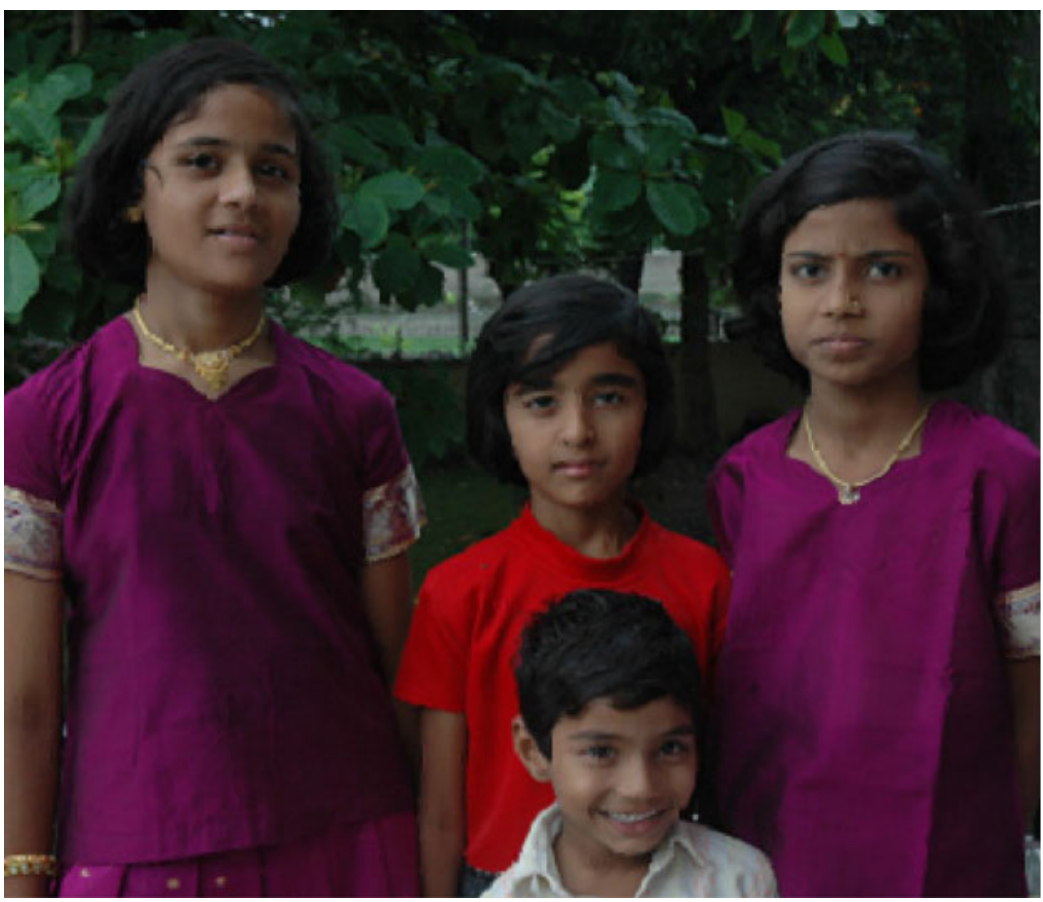

(b)

Figure 17. (a) Superimposed text on image. (b) Extracted image by proposed algorithm with PSNR $39.9043 \mathrm{~dB}$. 


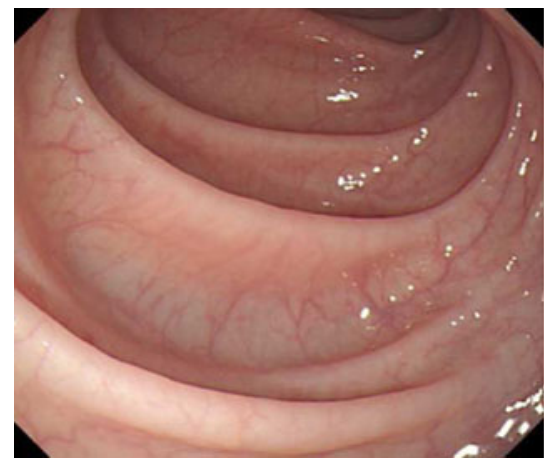

(a)

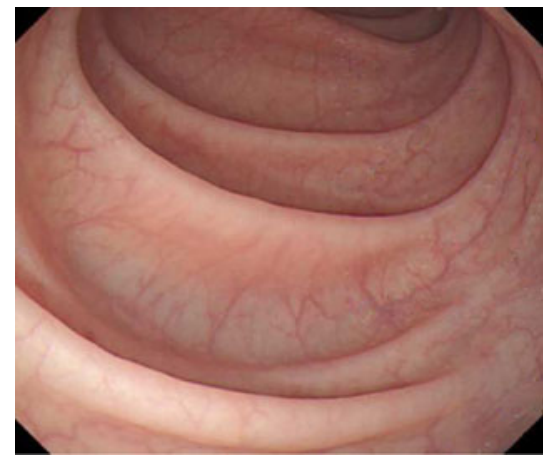

(b)

Figure 18. (a) Original endoscopic image. (b) Inpainted image by proposed algorithm.

experiments are conducted with both Bertalmio and FoE. Results prove that both these techniques are best suited for thin inpainting area and require large number of iterations to converge for thick inpainting area. For larger area inpainting, blur and blocky effects creep in.

This paper presents a new approach for image inpainting by propagating median information of the pixels from exterior of the inpainting area into the inpainting area. The results are better than both Bertalmio and FoE in the context of visual appearance and PSNR. Results are found to be better for both grey scale images as well as the colour images. The technique is also used successfully to remove artificial noise or scratches introduced in the images. Other applications of inpainting, viz. object removal, medical imaging, archaeological restoring are experimented with. The drawback of proposed technique is, that the images with texture background leave distinct edges around the mask area. Figure 19b shows a bad result obtained in which the connectivity between pipes (shown by red circles) is missing due to different textured background. Further, investigation is necessary to inpaint textured background.

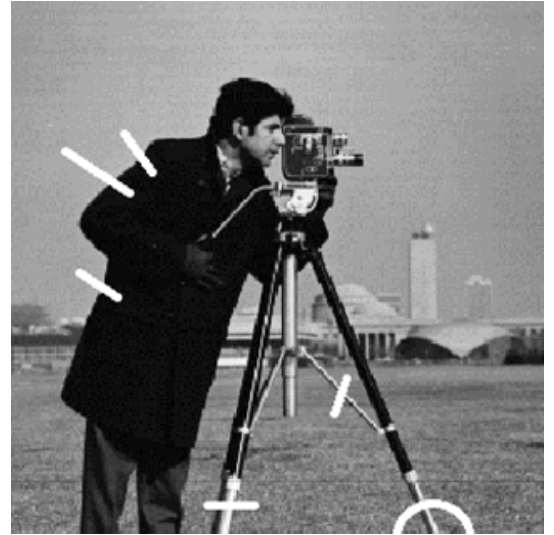

(a)

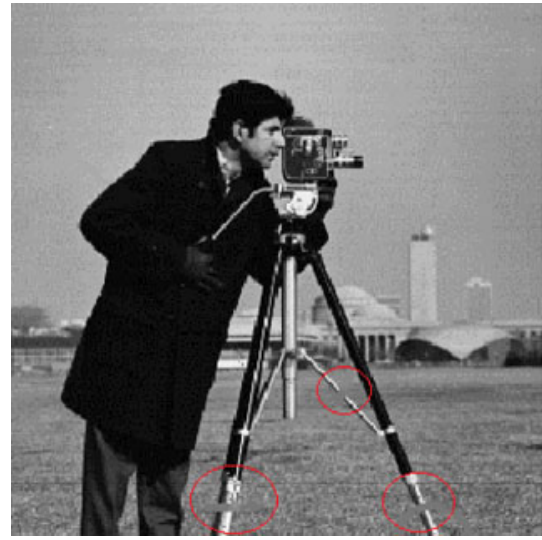

(b)

Figure 19. (a) Cameramen image with random small mask marked by white lines. (b) Result of inpainting in connectivity between pipes is missing. 


\section{Appendix A}

Median is the maximum likelihood estimator of location for Laplacian distribution (Ioannis \& Anastasios 1992).

$$
f(x ; \mu, \lambda)=\frac{1}{2 \lambda} e^{\frac{-|x-\mu|}{\lambda}} .
$$

This distribution is symmetric about $\mu$ and decreases exponentially to right and left, with $\lambda$ the dispersion parameter. It will be seen that the sample median $\bar{x}$ is the maximum likelihood estimator of $\mu$.

Proof: The dispersion parameter is given by

$$
\lambda=\sum_{i=1}^{n} \frac{\left|x_{i}-\bar{x}\right|}{n} .
$$

The likelihood function of $\mu$ is given by

$$
\phi=\frac{1}{(2 \lambda)^{n}} e^{-\frac{-\sum_{i=1}^{n}\left|x_{i}-\mu\right|}{n}} .
$$

Maximizing $\phi$ with respect to $\mu$ is equivalent to minimizing $\sum_{i=1}^{n}\left|x_{i}-\mu\right|$. This sum is minimum

for a set of $\left\{x_{i}\right\}$ when $\mu$ is the median of the set. Furthermore, setting $\partial \phi / \partial \lambda=0$ readily yields the estimator for $\lambda$.

\section{References}

Bertalmio M, Sapiro G, Caselles V, and Ballester C 2000 Image inpainting. In: Proc. SIGGRAPH. Computer Graphics Processing, 417-424

Bertalmio M, Bertozzi A L and Sapiro G 2001 Navier-Stokes, fluid dynamics and image and video inpainting. In: IEEE CVPR

Bertalmio M. Vese L, Sapiro G and Osher S 2003 Simultaneous strucure and texture image inpainting. In: UCLA CAM report $02-47$

Bianjing Bai, Zhenjiang Miao and Zhen Tang 2011 An improved structure propagation based image inpainting. In: Proc. SPIE 8009, 80091D

Chan F and Shen J 2000 Mathematical models for local deterministic inpainting. In: UCLA Computational and Applied Mathematics Reports 00-11, March

Chan F and Shen J 2001 Non-textured inpainting by curvature driven diffusion. J. Vis. Commun. Image. R. 12: 436-449

Chan F, Kang S H, Shen J 2002 Euler's elastica and curvature-driven diffusion. SIAM J. Appl. Math. 63(2): $564-592$

Cheng W-H, Hsieh C-W, Lin S-K, Wang C-W, Wu J-L 2005 Robust Algorithm for Exemplar-Based Image Inpainting. In: The International Conference on Computer Graphics, Imaging and Vision (CGIV 2005), 64-69, Beijing, China

Criminisi A, Perez P and Toyama K 2004 Object removal by exempler-based inpainting. IEEE Trans. Image Proc. 13(9): 1200-1212

Emile-Male G 1976 The restorer's handbook of easel painting. New York: Van Nostrand Reinhold

Efros A and Leung T 1999 Texture synthesis by non-parametric sampling. In: Proc. IEEE International Conference Computer Vision, 1033-1038, Corfu, Greece, September 
Grossauer H 2003 Digital Inpainting using the complex Ginzburg-Landau equation. In: Scale Space Method in Computer Vision, Lecturer Notes 2695

Grssauer H 2004 A Combined PDE and Texture Synthesis Approach to Inpainting, volume 3022, 214-224

Grossauer H and Scherzer O 2003 Using complex Ginzburg-Landau equation for image inpainting. In: Scale Space Method in Computer Vision, Lecturer Notes 2695

Hadhoud M M, Moustafa Kamel and Shenoda 2005 Digital image inpainting using modified convolution based method. Int. J. Sig. Process. Image Process. Pattern Recog. 1(1): 1-10

Hirani A and Totsuka T 1996 Combining frequency and spatial domain information for fast interactive image noise removal. In: Comput. Graph., SIGGRAPH, volume 96, 269-276

Ioannis Pitas and Anastasios N V 1992 Order statistics in digital image processing. In: Proc. IEEE, 18931921, December

Masnou S and Morel J M 1998 Level-lines based disocclusion. In: 5th IEEE International Conference on Image Processing, Chicago, IL, October 4-7

Noori H, Saryazdi S and Nezamabadi-pour H 2010 A convolution based image inpainting. In: 1st International Conference on Communication and Engineering, University of Sistan \& Baluchestan, December

Noori H, Saryazdi S and Nezamabadi-pour H 2011 A bilateral image inpainting. IJST Trans. Electr. Eng. 35(E2): 95-108, printed in the Islamic Republic of Iran, Shiraz University

Olivera M, Bowen B, Mckenna R and Chang Yu-Sung 2001 Fast Digital Image Inpainting. In: VIIP 2001, 261-266 [PUB]

Perona P and Malik J 1990 Scale-space edge detection using anisotropic diffusion. IEEE Transaction on Pattern Analysis 12(7): 629-639

Roth Stefan and Black Michael J 2005 Field of expert: A framework for learning image priors. In: IEEE Conference on Computer Vision and Pattern Recognition (CVPR), volume 2, 860-867, June

Tai X C, Osher S, Holm R 2006 Image inpainting using TV-Stokes equation. In: Image Processing Based on Partial Differential Equations, 473-482. Springer, Heidelberg

Telea A 2004 An image inpainting technique based on the fast marching method. J. Graphics Tools 9(1): 25-36. ACM Press

Walden S 1985 The ravished image. New York: St Martin's Press

Zhang Y, Pu Y-F, Hu J-R and Zhou J-L 2011 Fast X-ray CT metal artifacts reduction based on noniterative sinogram inpainting. In: Nucl. Sci. Symp. Med. Imaging Conf. (NSS/MIC)

Zhang Y, Pu Y-F, Hu J-R and Zhou J-L 2012 A class of fractional order variational image inpainting models. Appl. Math. Inf. Sc. Int. J. 2: 299-306 